\title{
Filarial worm (Loa loa) in the anterior chamber Report of two cases
}

\author{
OLABOPO OSUNTOKUN AND OYIN OLURIN \\ Department of Ophthalmology, University College Hospital, Ibadan, Nigeria
}

The adult Loa loa filarial worm wanders in the subcutaneous tissues of man and it is not uncommon for it to wander into the periorbital tissues and eyelids and often into the subconjunctival tissues where it can be seen moving rapidly across the conjunctival sac. It is because of such appearances in the conjunctival sac that it is often referred to as "the African eye worm". In such an environment Loa loa worms, though a nuisance, do not normally adversely affect vision.

Few cases of intraocular parasitism by the adult filarial worm have been reported in the literature (Wright, 1934; Nayar and Pillai, 1932; Chatterji, 1954; Mishra, 1958; Rose, 1966) and such reports document adult filaria (Wuchereria bancrofti) in either the vitreous or anterior chamber. This communication reports Loa loa filarial worms in the anterior chamber in two patients. We are not aware of any previous report of the Loa loa filarial worm in the anterior chamber in man.

Case r, a 22-year-old Nigerian woman, came to the Ophthalmology Clinic of University College Hospital, Ibadan, with a 6-months' history of pain, itching, and a sensation of "a worm eating away her right eye".

\section{Examination}

There was no perception of light in the right eye, but the eye was white and the cornea was translucent with discrete opacities. A live and active thread-like worm was seen in the anterior chamber. The light seemed to excite it and its movements became more vigorous, coiling and uncoiling and thrashing about. The anterior chamber appeared to be lined by a fibrous membrane which completely occluded the pupil.

Examination of peripheral blood samples for Microfilaria was negative. The total white cell count was 12,420/cu. mm. with eosinophilia 26 per cent., neutrophils 50 per cent., lymphocytes 22 per cent., and monocytes 2 per cent. Examination of a skin snip for Microfilaria was also negative. A general physical examination showed no abnormality. There was no lymphadenopathy. There were no palpable subcutaneous nodules. The chest and heart were normal and there was no hepatosplenomegaly.

Address for reprints: Olabopo Osuntokun, D.O., F.M.C.S. (Nig.), Department of Ophthalmology, University College Hospital, Ibadan, Nigeria.

\section{Treatment}

Since the eye was blind and appeared to be causing discomfort, it was excised under local anaesthesia and fixed in formol saline.

\section{Pathological report}

Macroscopical examination showed partial collapse of the anterior chamber, a hazy cornea, and total retinal detachment with subretinal gelatinous exudate. There was a worm in the anterior chamber which had the features of a male Loa loa.

Microscopical examination showed some vascularization of the peripheral cornea. The back of the cornea and the anterior surface of the iris and lens were lined by a fibrous membrane which occluded the chamber angle. The iris and ciliary body were oedematous, and the lens showed anterior subcapsular fibrosis. The retina was totally detached, disorganized, and degenerate, and showed neovascularization into the vitreous on one side. The subretinal space contained haemorrhagic albuminous exudate.

Case 2, a I5-year-old Nigerian schoolgirl, was referred to the Ophthalmology Clinic because of pain and feelings of worm movements in her right eye for 5 months, and blindness in the same eye for I month. She had received treatment with diethycarbamazine (banocide) and piriton for some days before she came to the hospital.

\section{Examination}

There was no perception of light in the right eye and the visual acuity in the normal left eye was $6 / 4$. The right eye showed slight conjunctival injection; the cornea was clear, and there was a very active worm in the anterior chamber (Figure). Minimal flare and cells were present in the anterior chamber. The lens was opaque.

\section{Laboratory investigations}

These included microscopical examination of thick and thin blood films. Skin snips showed no Microfilariae. The total white blood cell count showed i 4 per cent. eosinophilia.

\section{Treatment and progress}

The patient was asked to report a week later for extraction of the worm from the anterior chamber, because no bed 


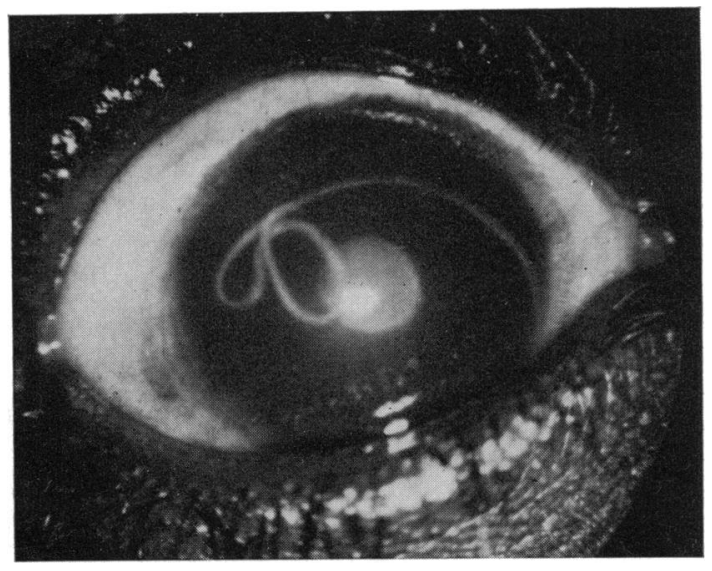

FIG. I Active Loa loa in anterior chamber

was available on the day she was first seen, but she defaulted. She returned 3 months later, when she complained of further pain and soreness in the affected eye. The worm was no longer mobile, the cornea was hazy, and the eye hypotensive. She was admitted to hospital and prepared for surgery to extract the worm from the anterior chamber.

\section{Operation}

The worm was found to be embedded in thick postinflammatory membrane and was adherent to the iris. This membrane had to be excised to gain access to the worm which had to be removed piecemeal. Postoperatively, there was a violent inflammation, periorbital oedema, and marked chemosis, and the cornea and anterior chamber became more hazy.

\section{Result}

She was given systemic antibiotics and steroids with little improvement, and the eye had to be enucleated 5 days later.

\section{Pathological report}

Histological examination of the worm removed showed pieces of adult Loa loa worm. The sex could not be determined.
The cornea was heavily pigmented. The specimen cut open showed some degree of choroidal detachment, and the retina was totally detached.

Microscopical examination showed a sutured penetrating corneal wound with lamellar necrosis and polymorphonuclear cell infiltration. The anterior chamber contained red blood cells and fibrin. There had been iridodialysis as well as supraciliary and suprachoroidal detachments. There were cataractous lenticular changes and a cyclitic membrane was associated with a funnelshaped retinal detachment. Subretinal exudation was prominent.

\section{Comment}

Infection with Loa loa is endemic in certain areas of Nigeria, especially in the Eastern region. Considering the frequency with which parasitaemia occurs in loasis, and the common occurrence of the worm in subconjunctival tissues, it is surprising that intraocular invasion by Loa loa is not more frequently seen. The route of entry into the eye is not known. The possibilities are that the full-grown worm penetrates the scleral coat of the eye or that the maturation of the worm from the larval form takes place inside the eye. Rose (I966) reported that the larval form probably emerged from a collarette vessel of the iris as an iris stromal defect was visible. Mishra (1958) also reported an intravitreal worm that broke loose from the macular area of the retina. The panophthalmitis which complicated removal of the worm from the damaged eye of our second patient was probably due to allergic reaction and secondary sepsis, although the patient did not benefit from steroid therapy. Therapy with diethylcarbamazine was not helpful either. Skin snips were examined for Microfilariae to exclude concurrent onchocercal skin infestation.

\section{Summary}

Two Nigerian patients with intraocular parasitism by adult Loa loa worms are reported. Whereas Loa loa infestation of the subcutaneous tissues around the eye usually has no permanent effect on visual acuity, in the two cases reported the affected eye became blind.

\section{References}

CHATterju, K. C. (1954) 7 . Indian med. Ass., 24, I 46

MISHRA, s. (1958) Arch. Ophthal., 6o, 945

NAYAR, K. K., and PILlAI, A. K. (I932) Brit. 7. Ophthal., r6, 549

ROSE, L. (1966) Arch. Ophthal., 75, I 3

WRIGHT, R. E. (1934) Brit. 7. Ophthal., 18, 646 